\title{
VIII.
}

\section{Ueber die römischen Handlungsbevolimächtigten.}

\author{
Von \\ Herrn E. Ruhstrat, \\ Präsidenten a. D. in Oldenburg.
}

Unter den Handlungsbevollmächtigten verstehe ich nicht die Institoren, welche meistens Sklaven oder Hauskinder des Geschäftsinhabers waren, sondern gewaltfreie Personen, die zur Betreibung eines Gewerbes als Procuratoren bestellt waren. Von einem derartigen Procurator ist z. B. die Rede in der aus Papinians lib. III resp. entlehnten 1. 19 pr. D. de institoria act. 14, 3, wo es heisst:

In eum, qui mutuis accipiendis pecuniis procuratorem praeposuit, utilis ad exemplum institoriae dabitur actio, quod aeque faciendum erit et si procurator solvendo sit, qui stipulanti pecuniam promisit.

Wäre der procurator in Fall dieser Stelle ein wirklicher institor, so hätte, wie sich nachher ergeben wird, die echte institoria gegeben werden müssen, da das Geschäft, welchem er vorgesetzt war, in verschiedenen Stellen als ein wirkliches Handelsgewerbe anerkannt wird. Die Contractschliessungen der Procuratoren aber fielen, wie ich hier gleich hervorheben will, $\mathrm{n}$ ich t unter das Edict des Prätors über die adjecticischen Klagen. Der dritte Contrahent erhielt auf Grund der Contracte, welche er im Interesse des Principals mit Procuratore n geschlossen hatte, die institorische actio neg. gestorum. Der Procurator haftete nicht und der Principal konnte die von dem Procurator begründeten Klagerechte ohne vorgängige Cession geltend machen, so dass also die rechtlichen Verhältnisse, welche durch die Contractschliessungen gewaltfreier Vertreter im Handelsverkehr begründet wurden, grosse Aehnlichkeit hatten mit denen, welche durch die jetzigen handelsrechtlichen Vertreter hervorgerufen werden. Doch bestand ein 
wesentlicher Unterschied. Dass im Interesse des Principals (contemplatione domini) contrahirt worden, ergab sich aus jeder bei der Contractschliessung erfolgten Bezugnahme auf die Vollmacht oder den Machtgeber, war also regelmässig ohne Schwierigkeit festzustellen. Nach den heutigen Grundsätzen muss der Vertreter entweder ausdrücklich oder stillschweigend im Namen des Vertretenen contrahiren. Wie schwierig und bedenklich aber die so oft nothwendige Feststellung ist, dass stillschweigend im Namen des Vertretenen contrahirt sei, und wie sehr die Betheiligten in dieser Hinsicht dem willkürlichen Ermessen der Richter anheimgegeben sind, will ich hier nicht wiederholen. - Es konnten nun zwar bei den Römern auch nach Einführung der institorischen actio neg. gestorum immer noch gewaltfreie Personen als Institoren bestellt werden, deren Contractschliessungen dann nach dem prätorischen Edict zu beurtheilen waren, aber das wird immer seltener vorgekommen sein, weil der Principal und der Vertreter sich besser dabei standen, wenn der letztere als procurator bestellt wurde. Freilich konnte der Institor bei jeder einzelnen Contractschliessung zu vereinbaren suchen, dass die Contractsklage gegen ihn selbst nicht erhoben werden dürfe, allein er hätte leicht das Misstrauen dritter Contrahenten erregt, wenn er jedesmal auf seine persönliche Sicherheit Bedacht genommen hätte. Auch wurde im Drange der Geschäfte nicht immer ängstlich erwogen, ob der Handel $\mathrm{zu}$ einem Streite führen werde. Darum musste es zweckmässiger erscheinen, dass die mit der Contractschliessung eintretende Haft des Vertreters regelmässig, ohne ausdrückliche Vereinbarung, gleich wieder aufhörte und nur bestehen blieb, wenn dies ausdrücklich verabredet wurde. Dass letzteres vorkam, ergiebt sich z. B. aus der schon angeführten und nachher noch näher zu besprechenden $1.19 \mathrm{pr}$. D. de instit. act. Statt dessen wurde in den Fällen, wo die alleinige Haft des Machtgebers dem Dritten nicht genügte, gleich bei der Contractschliessung auch wohl ein Bürge bestellt, was sich z. B. aus Papinians 1. 31 pr. D. neg. gest. ergiebt. Ulpian erwähnt in l. $10 \S 5$ D. mand. 17, 1 einen ganz ähnlichen Fall, in welchem Papinian sich darüber ausgesprochen hat, dass der mit der Aufnahme eines Darlehens beauftragte procurator 
einen Bürgen bestellt habe. Der Bürge gewährte dem Gläubiger die Sicherheit, welche der procurator nicht zu leisten brauchte und auch nicht leistete.

Ich will nun versuchen diese Aufstelluugen, soweit es erforderlich ist, aus den Quellen nachzuweisen. Den Zusammenhang der wohl zuerst von Papinian gegebenen actio ad exemplum institoriae ${ }^{1}$ ) mit dem prätorischen Edict über die adjecticischen Klagen habe ich in den Jahrbüchern für Dogmatik Bd. 26 S. $459 \mathrm{ff}$. zu entwickeln gesucht. Ich muss darauf kurz zurückkommen. Wenn ein institor ejus rei causa cui praepositus fuit mit einem Dritten contrahirte und der Dritte mit ihm darin einig war, dass ejus rei causa etc. contrahirt wurde, so wurde angenommen, dass der institor nicht bloss in eigenem Namen, sondern auch in dem des Principals contrahirt habe und es würde dem Dritten sein, dem institor gegenüber begründetes Rechtsmittel auch gegen den Principal gegeben, indem die im Namen des Principals erfolgte Contractschliessung als im voraus durch die Vollmacht gebilligt angesehen wurde. Die Rechtsmittel, welche der institor dem Dritten gegenüber begründet hatte, konnte der Principal ebendeshalb, weil auch der istitor haftete, in der Regel erst geltend machen, nachdem sie ihm vom institor cedirt waren. Ergab sich im einzelnen Falle, dass der institor bloss in eigenem Namen contrahirt hatte, so wurde angenommen, dass ein Rechtsverhältniss nur zwischen dem Dritten und dem institor begründet worden sei. Diese Vorschriften, welche für die Contracte der Institoren und Magister berechnet waren, fanden selbstverständlich auf die Contracte der Procuratoren keine Anwendung. Aber Papinian fand es unbedenklich, jene prätorische Auffassung der Vollmacht und ihrer rechtlichen Wirkung in Ansehung der von Bevollmächtigten geschlossenen Verträge, ausserhalb des Handelsrechts zur Geltung zu bringen und wusste unter Benutzung dieses Gesichtspunktes an der Hand des geltenden Rechts in den Fällen, wo ein blosser procurator ejus rei causa cui praepositus fuit mit einem Dritten contrahirt hatte, ein der institutoria an Wirkung gleichstehendes Rechtsmittel des Dritten gegen den

1) Vgl. Pernice, Labeo I S. 496. 
Machtgeber nachzuweisen, wobei er zugleich dahin gelangte, dass der procurator nicht in der Haft verblieb und der Machtgeber die vom procurator begründeten Klagen ohne Cession geltend machen konnte. Indem er die prätorische Auffassung, nach der einem institor, welcher ejus rei causa etc. contrahirte, ein doppelter Vertragswille zuzuschreiben war, bei Seite liegen liess, vielleicht deshalb, weil er eine analoge Anwendung dieser Bestimmung für unzulässig hielt, daneben aber der Meinung war, dass die prätorische Vorschrift dem Parteiwillen selten entspreche, begründete er sein neues Rechtsmittel etwa in folgender Weise: Wenn der Procurator mit dem Dritten bei der Contractschliessung darin einig ist, dass vollmachtgemäss, also lediglich wegen des Machtgebers und in dessen Interesse contrahirt werde, so müssen sie vernunftgemäss den Willen haben, dass die in der Person des Procurators entstehenden Verbindlichkeiten lediglich von dem Machtgeber erfüllt werden sollen. Das entspricht ja allerdings nicht der hergebrachten Schulmeinung, aber es entspricht und entsprach schon zu Papinians Zeit dem Parteiwillen. Danach verzichtet der Dritte gleich mit der Contractschliessung contemplatione domini auf sein gegen den Vertreter begründetes Rechtsmittel, während dieser den Verzicht annimmt und zugleich kraft seiner Vollmacht im Namen des Principals genehmigt, indem er stillschweigend erklärt, dass dieser zu erfüllen habe. Kann der Institor den Principal dadurch verpflichten, dass er auf Grund seiner Vollmacht im Namen desselben contrahirt, so erscheint die Annahme nicht bedenklich, dass der Procurator den Machtgeber auf Grund der Vollmacht in der Weise verpflichten kann, dass er in dessen Namen genehmigt. Wer das nicht zugeben will, der muss auch in dem Fall, wo der Bevollmächtigte in eigenem Namen contrahirt, dabei aber mit Dritten ausdrücklich vereinbart hat, dass der Machtgeber zu erfüllen habe, dem Dritten jedes Rechtsmittel absprechen und zwar aus dem Grunde, weil nicht die richtige Form gewählt sei. Wollte aber ein Machtgeber sich darauf berufen, so würde er damit beweisen, dass er sich auf Schleichwegen seiner unleugbaren Verbindlichkeit zu entziehen suche, denn ob ein vollmachtgemäss abgeschlossenes Geschäft im Namen des Principals errichtet 
wird, oder in der Weise, dass der Vertreter in eigenem Namen contrahirt, daneben aber dem Machtgeber kraft seiner Vollmacht die Verbindlichkeit auflegt, die Erfüllung des Contracts zu übernehmen, kann für die unbefangene Beurtheilung keinen Unterschied machen. Die Meinung aber, dass die einmal in der Person des Vertreters entstandene Verbindlichkeit sich nicht durch blosse Vereinbarung übertragen lasse, ist als verfehlt zu betrachten. Die Uebertragung erfolgt, wie ich schon früher gezeigt habe, in der Weise, dass der Gläubiger contemplatione domini und mit der Absicht vom Machtgeber Ersatz zu fordern, auf seine Contractsklage verzichtet und dass der Vertreter seiner mit dem Gläubiger getroffenen $\mathrm{Ab}$ rede entsprechend, die neg. gestio des Gläubigers im Namen des Machtgebers genehmigt. Papinian nannte die Klage daher actio neg. gestorum und zwar mit dem Zusatz: ad exemplum institoriae, weil sie wie die institoria auf der Vollmacht beruhte. - In Ansehung des Beweises dieser Auffassung Papinians will ich mich hier, unter Bezugnahme auf meine früheren Ausführungen kurz fassen, soweit ich nicht neue Argumente anzuführen habe. Es kommt hier vorzugsweise in Betracht die 1. 31 pr. D. neg. gest. 3,5, in der Papinian den Namen der Klage vollständig angiebt. In dem hier von ihm behandelten Fall contrahiren der Mandatar und der Gläubiger mit Bezugnahme auf das dem letzteren mitgetheilte Schreiben des Mandanten (cujus litteras secutus creditor contraxit). Daraus wird von Papinian ohne weiteres gefolgert, dass dem Gläubiger die actio neg. gestorum ad exemplum institoriae gegen den Machtgeber zusteht, wonach demselben die Contractsklage gegen den Bevollmächtigten (Mandatar) nicht zusteht, indem die actio neg. gestorum nur durch den Verzicht auf die Darlehensklage ins Leben treten konnte. Die Contrahenten waren darin einig, dass wegen der Vollmacht und des Machtgebens contrahirt werde und machten damit aus, dass die contractliche Verbindlichkeit von dem Machtgeber $\mathrm{zu}$ erfüllen sei, was damit zugleich von dem Vertreter kraft seiner Vollmacht im Namen des Machtgebers genehmigt wurde. So viel ich weiss, ist Baron, adjecticische Klagen S. 193, der einzige Schriftsteller, welcher sich nicht damit begnügt, die institorische actio neg. gestorum für die adjecticische actio quasi 
institoria zu erklären. Ich will darum über seine Ansicht hier noch Einiges bemerken. Er sagt: Papinians Auffassung in 1. 31 pr. D. neg. gest. 3, 5 sei die, dass man durch Gewährung eines Darlehens eine doppelte Obligation contrahiren könne, nämlich die Darlehensobligation gegenüber dem contrahirenden Vertreter und die Geschäftsführungsobligation gegenüber dem Geschäftsherrn. Dabei wird auf S. 41 der Abhandlung verwiesen, wo es heisst: „Ich kann Jemandem ein Darlehen geben mit der Abrede, dass es zum Besten eines Dritten verwendet werde. Indem der Darlehnsgeber diese Abrede zum Bestandtheil des Vertrages erhebt, gerirt er die Geschäfte des Dritten und erwirbt gegen ihn eine Klage nach den Grundsätzen der negotiorum gestio." Dazu bemerke ich Folgendes: Wenn Jemand dem, in eigenem Namen handelnden, Stellvertreter eines Anderen Geld leiht, so bekommt er für sein Geld die Darlehensklage, wonach er nichts aufopfert und wenn er das Darlehen mit einseitiger contemplatio domini gegeben hat, so kann diese contemplatio ihm die actio neg. gestorum nicht begründen, weil sie keine Aufopferung zur Folge hat, da er trotz derselben die Darlehensklage gegen den Stellvertreter anstellen kann. Sollte mit der Darlehensklage nichts zu holen sein, so wäre das die Folge der Besorgung seines eigenen Geschäfts. Wenn aber bei der Contractschliessung zugleich die Abrede getroffen wird, dass der geliehene Betrag zum Besten des Geschäftsherrn verwendet werden soll, so erklärt der Darlehensgeber im Einverständniss mit dem Stellvertreter: „Der geliehene Betrag, welcher nach unserer Abrede für den Machtgeber verwendet werden soll, braucht eben desshalb nicht an mich zurückgezahlt zu werden; ich gebe meinen Anspruch contemplatione domini auf und werde meine Entschädigung direct von dem Machtgeber mit der actio neg. gestorum fordern. Deine Sache aber ist es, den vollmachtsgemäss geliehenen Betrag vollmachtsgemäss zu verwenden, denn jene Abrede hat durchaus nicht den Sinn, dass der geliehene Betrag in meinem Auftrage von Dir verwendet werden soll"1). Mit der Abrede, dass der Betrag zum Besten des Machtgebers, also vollmachtgemäss zu verwenden sei,

1) A. M. ist Wendt in den Jahrb. für Dogmatik B. $28 \mathrm{~S} .15 \mathrm{ff}$. 
erklärten beide Theile sich darin einverstanden, dass der Machtgeber dem Gläubiger Ersatz zu leisten laabe, womit der Stellvertreter zugleich im Namen des Machtgebers, kraft der von diesem gegeben Vollmacht, genehmigte.

In Ansehung der 1. $10 \$ 5$ D. mandati 17, 1 bemerke ich noch, dass Papinian hier nach Ulpians Bericht denselben Fall behandelt, wie in 1.31 pr. D. neg. gest. ${ }^{1}$. Daraus ergiebt sich, dass er unter der actio quasi institoria keine andere Klage versteht, als die in 1.31 pr. gegebene actio neg. gestorum quasi institoria ${ }^{2}$ ). Dass Ulpian den Papinian missverstanden habe, braucht also nicht vermuthet $\mathrm{zu}$ werden.

In $1.6 \S 1$ (l. $5 \S 3)$ D. neg. gest. 3,5 behandelt Ulpian den schon oben besprochenen Fall, wo einem procurator Geld geliehen ist mit der Abrede, dass der geliehene Betrag zum Besten des Machtgebers zu verwenden sei. Ulpian spricht sich bestimmt dahin aus, dass der Gläubiger den procurator, mit welchem er contrahirte, $\mathrm{nicht}$ verklagen, gegen den Principal aber die actio neg. gestorum anstellen kann.

In $1.5 \$ 1$ D. quando ex facto tutoris 26,9 sagt $\mathrm{Pa}-$ pinian mit deutlichen Worten, dass der Gläubiger, welcher einem Vormund Geld geliehen hat mit der Abrede, das Geld zum Besten des Pupillen (nämlich zur Bezahlung einer Judicatsschuld) zu verwenden, den Vormund nicht verklagen kann.

In $1.13 \& 25$ D. de act. emti vend. 19, 1 berichtet Ulpian über einen von Papinian (lib. III resp.) behandelten Fall, in welchem ein procurator contemplatione domini mit einem Dritten contrahirt (einen Kaufcontract geschlossen) hat. Papinian und Ulpian sind der Meinung, dass der Käufer die utilis actio ad exemplum institoriae gegen den Machtgeber anstellen und dass letzterer eben desshalb auch umgekehrt mit der Contractsklage gegen den Käufer auftreten kann. Schon diese zuletzt erwähnte Annahme liefert dell Beweis, dass die Juristen unter der actio ad exemplum institoriae actionis keine andere Klage verstehen können als die actio neg. gestorum ad exemplnm institoriae actionis.

1) Das meint auch Baron S. 194. - 2) Vgl. darüber, dass Papinian den Namen der Klage nicht vollständig angiebt, die Schlussbemerkung. 
Ueber 1. 5 Cod. de exerc. et instit. 4, 25:

Si pecuniam mutuam accipere Demetriano Domitianus mandavit et hoc posse probare confidis, ad exemplum institoriae eundem Domitianum apud competentem iudicem potes convenire

bemerke ich folgendes. Der Fall, in welchem die Kaiser um Entscheidung gebeten waren, mag etwa so gelegen haben: Demetrianus hatte eine bestimmte Summe von dem Bittsteller geliehen und zwar unter der Behauptung, dass er von Domitianus beauftragt sei, das Geld zu leihen. Zugleich hatte er dem Darleiher bescheinigt, dass er im Auftrage des Domitianus die entsprechende Summe geliehen erhalten habe. Die Kaiser entscheiden nun, dass dem Gläubiger die actio (neg. gestorum) ad exemplum institoriae gegen Domitian zustehe, wenn er den von dem letzteren geleugneten Auftrag sollte beweisen können. War der behauptete Auftrag nämlich in der That gegeben, so lag die Sache gerade so, wie in dem Fall der 1. 31 pr. D. neg. gest. 3, 5. Zwar meint Baron S. 135, das Rescript liabe die Argumentation der 1.31 pr. aufgegeben, da es von einer negotiorum gestio nichts wisse, allein in jedem Falle, wo nach Papinian die actio ad exemplum institoriae oder die actio quasi institoriae gegeben wird, versteht er darunter die actio negot. gestorum ad exemplum institoriae actionis. Dasselbe thun die Kaiser im Fall der 1.5 Cod. cit.

In 1.6 Cod. eod. sagen die nämlichen Kaiser (Diocletian und Maximian):

Qui secutus domini voluntatem cum servo ipsius habuit contractum, ad instar actionis institoriae recte de solido dominum convenit.

Unter der actio ad instar institoriae verstehen sie die actio neg. gostorum ad exemplum institoriae actionis. Diese Klage unterschied sich darin von den Stellvertretungsklagen ${ }^{1}$ ), dass es nicht darauf ankam, ob die Mittelsperson mit der kundgegebenen Absicht contrahirt habe, als Stellvertreter zu handeln und verdiente in manchen Fällen schon aus diesem Grunde den Vorzug.

1) Vyl. Savigny, Obligationen Bd. 2, S. 33. 
Alle diese bis jetzt angeführten Gesetze, welche sich über die actio ad exemplum institoriae aussprechen, habe ich hier insbesondere desshalb wieder zusammengestellt, weil sie den Beweis, welchen ich jetzt noch in Ansehung der Handlungsprocuratoren beibringen werde, ganz wesentlich unterstützen.

Was nun das römische Handelsrecht betriftt, so konnte Papinian insofern nicht eingreifen, als ilim die prätorischen Bestimmungen im Wege standen, insbesondere der Grundsatz, nach weichem dem contrahirenden Institor oder Magister regelmässig ein doppelter Vertragswille zugeschrieben wurde, was eine doppelte Obligation zur Folge hatte. Aber Papinian glaubte annehmen zu dürfen, dass die prätorischen Bestimmungen auf die Contractschliessungen der Procuratoren des Handelsverkehrs nicht anzuwenden seien ${ }^{1}$ ). War eine gewaltfreie Person zur Betreibung eines Handelsgewerbes als procurator bestellt, so waren der Principal und der procurator darin einig, dass die Contractschliessungen dieses procurator nicht den prätorischen Bestimmungen, sondern den abgesehen davon geltenden Vorschriften unterstellt werden sollten und es wurde auf Grund solcher Contracte seit $\mathrm{Pa}$ pinian die institorische actio neg. gestorum gegeben ${ }^{2}$ ). Uebrigens glaube ich auf Grund der mitgetheilten l. 5 Cod. de exerc. et inst. annehmen zu müssen, dass die genannte Klage, jedenfalls zur Zeit der erwähnten Kaiser, mitunter auch in solchen Fällen gegeben ist, wo jemand mit einem institor oder magister - derselbe mochte gewaltfrei sein oder nicht contrahirt hatte. Zwar wurde solchen Vertretern regelmässig ein doppelter Vertragswille zugeschrieben, aber diese Annahme traf thatsächlich nicht immer zu. Wenn sich im einzelnen Fall herausstellte, dass der Institor bloss im eigenen Namen contrahirt habe, aber doch mit dem Dritten darin einig gewesen sei, dass lediglich im Interesse des Principals, also wegen des vollmachtgemässen Zwecks (propter domini voluntatem) contrahirt werde, so war die actio institoria nicht begründet, wohl aber die actio neg. gestorum ad exemplum in-

1) Der procurator war durchgängig Ersatzmann. So auch Pernice, Labeo Bd. 1 S. $495 . \quad$ - $\left.{ }^{2}\right)$ War dem dritten Contrahenten nicht mitgetheilt, dass die im Geschäft angestellte Person ein procurator sei, so galt dieselbe als institor. Dies folgt 2. B. aus 1.18 D. de instit. 14, 3 . 
stitoriae. Dass der Gläubiger in derartigen Fällen eine Klage gegen den Machtgeber hat, entspricht doch wahrlich auch dem Rechtsgefühl und der Billigkeit. Gleichwohl ist man der Meinung, dass nach den heutigen Grundsätzen in derartigen Fällen eine Klage des Dritten nnr gegen den Vertreter begründet sei. Hat der Vertreter bloss in eigenem Namen contrahirt, daneben aber mit dem Dritten ausdrücklich vereinbart, dass die von ihm übernommene Verbindlichkeit von dem Machtgeber $z u$ erfüllen sei, so glaubt man den Dritten auf die Klage gegen den Vertreter beschränken zu müssen, und die Motive zu dem Entwurf eines bürgerlichen Gesetzbuchs für das deutsche Reich wollen diese bedenkliche Consequenz sogar mit in die Zukunft hinübernehmen! Nach meiner Auffassung ist es eine ganz unzulässige Bevormundung des Publikums und eine unnöthige, bei den Römern schon durch Papinians neue Klage abgeschaffte Beschränkung der Bewegungsfreiheit, wenn man vorschreibt, dass eine Klage dritter Contrahenten gegen die Machtgeber nicht durch freie Vereinbarung der Dritten mit den Bevollmächtigten begründet werden kann, sondern nur dadurch, dass von Seiten der Bevollmächtigten im Namen der Machtgeber contrahirt wird.

Es braucht kaum bemerkt zu werden, dass es lediglich von dem Principal abhing, welche Machtbefugniss er den von ihm bestellten Procuratoren einräumen wollte. Der mit der Betreibung eines Handelsgewerbes beauftragte Procurator hatte im wesentlichen dieselbe Vollmacht wie ein institor. Es gab indess Procuratoren mit geringeren Befugnissen ${ }^{1}$ ). Es kam auch der umgekehrte Fall vor. Der Procurator konnte unter Umständen sogar Institoren für den Principal anstellen ${ }^{2}$ ) und mit (ler actio institoria verklagt werden ${ }^{3}$ ).

Ich wende mich nun zu den Stellen, welche sich speciell auf die Handelsprocuratoren beziehen.

1) Die gleich im Anfang erwähnte und abgedruckte 1.19 pr. D. de instit. 14, 3 von Papinian. Ich habe früher mit Baron S. 190, 193 annehmen zu müssen geglaubt, dass $\mathrm{Pa}-$ pinian das hier in Frage stehende Gewerbe nicht als ein eigent-

1) Vgl. z. B. $1.5 \$ 10$ D. de instit. $\left.14,3 .-{ }^{2}\right)$ L. 5 \$ 18 D. eod. 3) L. 6 eod. 
liches Handelsgewerbe habe gelten lassen, vielleicht wegen der Seltenheit des Geschäfts, dass er denjenigen qui mutuis accipiendis pecuniis praepositus est, darum nicht als wirklichen institor anerkannt und auf Grund seiner Contractschliessungen eine actio quasi institoria gegeben habe. Diese Auffassung ist nicht haltbar, denn es ergiebt sich aus anderen Stellen mit Sicherheit, dass das fragliche Geschäft als ein wirkliches Handelsgewerbe anerkannt ist. Zunächst sagt Papinian selbst in $1.19 \S 1$ eod., dass der servus, welchen Jemand apud mensam pecuniis accipiendis bestellt hat, ein wirklicher institor ist. Ferner bemerkt Ulpian in l. 13 pr. eod.:

Habebat quis servum merci oleariae praepositum Arelate. Eundem et mutuis pecuniis accipiendis; acceperat mutuam pecuniam. Putans creditor, ad merces eum accepisse, egit proposita actione; probare non potuit, mercis gratia eum accepisse; licet consumta est actio, nec amplius agere poterit, quasi pecuniis quoque mutuis accipiendis esset praepositus, tamen Iulianus, utilem ei actionem competere, ait.

Wurde auf Grund der Contractschliessung eines Sklaven der mutuis accipiendis pecuniis angestellt war, die echte institoria gegeben, was nach dieser Stelle nicht zweifelhaft ist, so wäre sie auch gegeben, wenn statt des Sklaven eine gewaltfreie Person bestellt gewesen wäre, denn ob der institor frei war oder nicht, machte keinen Unterschied ${ }^{1}$ ). In dem Fall der Stelle wird freilich eine utilis institoria gegeben, aber nur darum, weil die echte institoria consumirt war. Abgesehen von der Consumtion hält Ulpian die echte institoria für begründet. Warum wird denn nun diese in dem Fall der 1.19 pr. nicht gegeben, obgleich hier von dem nämlichen Gewerbe gehandelt wird, wie in 1.13 pr.? Ich antworte darauf: weil im Fall der l. 19 pr. ein procurator, kein institor, präponirt war und die prätorischen Grundsätze nicht auf die Contractschliessungen der Procuratoren angewandt wurden ${ }^{2}$ ). Die

1) Vgl. $1.7 \$ 1$ D. de instit. 14,3. $\left.-{ }^{2}\right)$ Da ein wirkliches Handelsgewerbe in Frage stand, so ist es doch sicher auffallend, dass $\mathrm{Pa}$ pinian sagt, es sei ein procurator bestellt. Die Meinung, das jeder gewaltfreie institor als procurator bezeichnet sei, Kritz Pandekten I, 1, S. 340, ist nicht halthar. Vgl. z. B. I. $7 \$ 1$ eod. 
ausdrückliche oder stillschweigende Vereinbarung des procurator mit dem dritten Contrahenten, dass wegen des Principals, also in dessen Interesse contrahirt wurde, legte Papinian aber dahin aus: dass die lediglich im Interesse des Principals vom procurator übernommene Verbindlichkeit auch lediglich von Principal zu erfüllen sei. Aus $1.19 \mathrm{pr}$. ergiebt sich nun in der That, dass der procurator aus dem mutuum nicht verklagt werden kann. Wäre nämlich die Darlehensklage begründet gewesen, so hätte Papinian mit den Worten ausgereicht: quod aeque faciendum erit et si procurator solvendo sit. Die Erwähnung der Stipulation wäre überflüssig gewesen, denn ob der procurator auf Grund des Darlehens oder einer damit verbundenen Stipulation verklagt werden konnte, war gleichgültig, wenn es Papinian darauf angekommen wäre, die adjecticische Doppelhaft zum Ausdruck zu bringen und festzustellen, dass der Principal nicht bloss in subsidium hafte ${ }^{1}$ ), was umsoweniger Papinians Meinung gewesen sein kann, als seit Einführung der a. exercitoria und institoria gerade in entgegengesetzter Richtung, nämlich darüber Zweifel entstanden zu sein scheinen, ob neben der adjecticischen Klage nun auch noch die Klage gegen den Vertreter stattfinde. Darauf scheint Paulus in $1.5 \& 1 \mathrm{D}$. de exercitoria act. 14,1 hinzudeuten :

- - Nam et cuivis alii non obstat hoc edictum, quominus cum magistro agere possit; hoc enim edicto non transfertur actio, sed adjicitur.

Auf die Frage, welche Bedeutung man hiernach der von Papinian in 1.19 pr. erwähnten Stipulation beizulegen habe, ist Folgendes zu antworten: Die von Papinian gegebene utilis ad exemplum institoriae actio beruhte darauf, dass der Gläubiger seinen dem procurator gegenüber begründeten Darlehensanspruch contemplatione domini aufgegeben hatte, mit der $\mathbf{A b}$ sicht von dem Principal Ersatz zu fordern und diese negotiorum gestio als mittels der Vollmacht genehmigt anzusehen war. Aber daraus folgte nicht, dass die Klage, etwa aus dem Grunde, weil sie formell eine actio neg. gestorum, also eine Entsclıädigungsklage war, nur stattfinde, wenn der Gläubiger

1) Dies wird angenommen von Mühlenbruch, Gession $§ 14$ Note 287. 
sonst überall keinen Ersatz würde erhalten können. Wie der Procurator eine vierte Person als Bürgen der Schuld des Principals hätte stellen können, was Papinian in 1.31 pr. D. neg. gest. 3, 5 ausdrücklich hervorhebt, so konnte er auch sich selber durch Stipulation verpflichten, den Betrag, weichen der Principal schuldig geworden war zu zahlen, ohne der institorischen actio neg. gestorum damit ihre Grundlage zu entziehen. - Der in $1.6 \S 1(1.5 \S 3)$ D. neg. gest. behandelte Fall liegt in Betreff der Stipulation anders. Der procurator hat sich durch Stipulation verpflichtet, seine eigene Darlehensschuld zu bezahlen und es ist diese Verbindlichkeit, nebst der aus dem Darlehen, durch die Nebenberedung weggefallen, dass der geliehene Betrag vollmachtgemäss verwendet, also nicht. an den Gläubiger zurückgezahlt werden soll. Im Fall der 1.19 pr. hat der procurator sich durch Stipulation verpflichtet, die Schuld des Principals aus neg. gestio zu bezahlen, so dass der Gläubiger nun wieder zwei Schuldner hat, nämlich den procurator aus der Stipulation und den Principal aus der negotiorum gestio.

2) Nachdem Papinian in 1.19 pr. den Fall behandelt hat, wo einem wirklichen Handelsgewerbe eine gewaltfreie Person als procurator vorgesetzt ist, dessen Contractschliessungen den Principal formell in anderer Weise verbindlich machen, als die eines institor, indem sie die actio neg. gestorum ad exemplum institoriae gegen ihn begründen, geht er in $\S 1$ auf den Fall über, wo Jemand einem gleichen Handelsgewerbe seinen Sklaven als institor vorgesetzt hat, dem er im Laufe der Geschäftsführung die Freiheit giebt, und bemerkt darüber, dass die Freilassung des institor die causa der Haft des Principals nicht verändere. Er sagt nämlich

Si dominus, qui servum institorem apud mensam pecuniis accipiendis habuit, post libertatem quoque clatam, idem per libertum negotium exercuit, varietate status non mutabitur periculi causa.

Es kann wohl nicht zweifelhaft sein, dass Papinian damit Folgendes aussprechen will: „ein Sklave, der als institor angestellt ist, wird durch die im Laufe der Verwaltung erfolgende Freilassung nicht $z$ u einem procurator; die Contracte, welche er als Freigelassener schliesst, verpflichten 
den Principal also nicht in anderer Weise, als die welche er vor der Freilassung geschlossen hat." Die Gewaltfreiheit eines handelsrechtlichen Vertreters beweist noch nicht, dass derselbe ein procurator ist. Dritte Contrahenten, welchen die Freilassung bekannt geworden war, hatten keinen Grund bloss darum anzunehmen, dass der institor nun nicht mehr institor, sondern procurator sei. - Wenn man bei Erklärung der 1.19 $\S 1$ ganz absieht von dem, was Papinian eben vorher gesagt hat, so fragt man sich vergeblich, wie denn darüber ein Zweifel entstehen konnte, dass ein freier institor den Principal in der nämlichen Weise verpflichte, wie ein unfreier und wie Papinian dazu gekommen sei, etwas ganz Selbstverständliches auszuführen. Da nämlich der freie institor ebenso wie der unfreie, mittelst seiner Contracte die echte institoria begründete, so verstand es sich ganz von selbst, dass die causa periculi durch die Freilassung des institor keine Veränderung erlitt.

3) In 1. 16 D. de institoria 14,3 ist von einem villicus die Rede, der mercibus distrahendis praepositus est. Derselbe ist nach meiner Auffassung kein institor, sondern ein procurator, weshalb auf Grund seiner Contracte eine Klage ,exemplo institoriae" gegeben wird. Paulus sagt hier:

Si cum villico alicujus contractum sit, non dabitur in dominum actio, quia villicus propter fructus percipiendos, non propter quaestum praeponitur. Si tamen villicum distrahendis quoque mercibus praepositum habuero, non erit iniquum, exemplo institoriae actionem in me competere.

Wer präponirt war ad mercium distractionem galt regelmässig als institor ${ }^{1}$ ). Wie beim Handwerk $1.6 \$ 5$ eod., so kam der institor auch in der Landwirthschaft vor und wenn der villicus in dem Fall der 1. 16 ein Sklave gewesen wäre ${ }^{2}$ ), so hätte Paulus jedenfalls die actio institoria gegeben. Da er anstatt derselben die actio exemplo institoriae giebt, so muss angenommen werden, dass er den gewaltfreien villicus als

1) Vgl. 1. 5 \$ 12 D. de instit. 14,3. $-{ }^{2}$ ) Dass nicht jeder villicus ein Sklave war, erhellt z. B. aus $1.18 \S 4$ D. de instruct. vel instrum. leg. 33,7 . 
einen mit dem Verkauf der Erndte beauftragten procurator beurtheilt hat. Der villicus war entweder als institor oder als procurator angestellt, aber hätte der Principal ihn als institor präponirt, so würde Paulus die echte institoria gegeben haben. Zur Gewährung einer adjecticischen actio quasi institoria hatte er jedenfalls gar keine Veranlassung.

Die vorstehende Ausführung dürfte zur weiteren Bestätigung meiner Ansicht über die institorische actio neg. gestorum dienlich sein, doch sind die Beweise damit noch nicht abgeschlossen. Ich hoffe später noch weitere beibringen zu können, will aber in dieser Beziehung schon hier auf folgenden Gesichtspunkt hinweisen. Man nimmt gewöhnlich an ${ }^{1}$ ), dass seit Papinian aus jeder von einem Bevollmächtigten ohne Grenzüberschreitung angeknüpften obligatio, dem welcher mit Rücksicht auf den Geschäftsherrn contrahirt hatte, nach der Analogie (ler institorischen Klage, eine utilis actio ad exemplum institoriae, oder quasi institoria, gegeben sei. Es soll auch hier, wie bei der echten actio institoria, lem dritten Contrahenten seine gegen den Bevollmächtigten begründete Klage utiliter, als actio adjectitiae qualitatis, gegen den Geschäftsherrn eingeräumt sein und zwar zuerst von Papinian, weshalb man ihn als den Schöpfer der actio quasi institoria bezeichnet. Dabei erhebt sich indess die Frage, ob Papinian sich fiur befugt erachten konnte, geradezu über den Kreis hinauszugehen, welchen der Prätor ausdrücklich für die adjecticischen Klagen festgesetzt hatte. Nach meiner gegenwärtigen Auffassung ${ }^{2}$ ) hat er diesen Kreis nicht überschritten, indem die von ihm gegebene und auch wohl eingeführte actio quasi institoria iberall keine adjecticische Klage ist. Er hat nur die rechtliche Bedeutung, welche der Vollmacht in Beziehung auf die Contracte der Institoren und Magister, sowie der Haussöhne, welche auf Grund eines jussus handelten, vom Prätor beigelegt war, verallgemeinert und unter Verwerthung dieses Gesichtspunkts, aus den geltenden Rechtsgrundsätzen eine actio neg. gestorum hergeleitet, welche der actio

1) Man vgl. z. B. Mühlenbruch, Cession S. 142. 143. - ${ }^{2}$ ) In den Jahrb. für Dogm. Bd. 26 S. 468 war ich mit Rücksicht auf 1.19 pr. D. de instit. 14, 3 noch anderer Meinung, während ich diese Stelle nach dem Obigen jetzt ganz anders verstehe. 
institoria an Wirkung gleichkam, da sie stattfand ,esti pecunia non sit in rem ejus versa" 1 ). Papinian hatte aber auch umsomehr Veranlassung gerade in dieser Weise einzugreifen, als er dadurch zu Ergebnissen kam, welche denen der prätorischen Einrichtung weit vorzuziehen waren. Fr gelangte um es kurz zu wiederholen dahin, dass der procurator, welcher contemplatione domini mit einem Dritten contrahirt hatte, nicht in der Haft blieb, dass der Machtgeber sofortige Berechtigung in Ansehung der von dem procurator begründeten Rechtsmittel erhielt und dass die Contractschliessung nicht im Namen des Principals erfolgt zu sein brauchte. - Die in 1. 31 pr. D. neg. gest. 3, 5 von Papinian gegebene actio neg. gestorum ad exemplum institoriae actionis, welche er auch kürzer als actio quasi institoria oder actio ad exemplum institoria bezeichnet, hätte eigentlich schon längst darauf aufmerksam machen sollen, dass die Sache sich nicht in der Weise verhalten kann, wie man anzunehmen pflegt. Denn wie köunte Papinians actio neg. gestorum eine adjecticiscbe Klage sein! Aber trotzdem erklärt selbst Mühlenbruch, einer der gründlichsten Kenner und Bearbeiter dieser Rechtsverhältnisse, die actio neg. gestorum Papinians für identisch mit der adjecticischen actio quasi institoria, womit er aber selbstverständlich nichts beweist. - Die Stellen, in welchen Papinian von der neuen Klage handelt, sind alle aus dem 3. Buch oder doch aus dem 2. und 3. Buche der Responsen ${ }^{2}$ ). Dass er hier ihren langen Namen nicht immer vollstündig wiederholt, ist natürlich und konnte von denen, welche die Responsen lasen, nicht missverstanden werden ${ }^{3}$ ).

\section{Nachtrag.}

Die vorstehende Arbeit war schon vollendet, als mir die in den Jahrbuichern für Dogmatik, B. 28, S. 287 ff. mitgetheilte

1) L. 31 pr. D. neg. gest. 3, 5. Sie beruhte, wie die institoria, auf der Vollmacht und darin bestand die Aehulichkeit dieser Klagen, sowie der Grund, aus welchem Papinian seine neue Klage actio quasi institoria oder ad exemplum institoriae nennt. - 2) Nach Pernice S. 496 N. 5 stammen sie alle aus dem 3. Buch. - 3) Späterhin ist es freilich stets missverstanden, weil man die Responsen nicht ganz vor sich hatte. 
Abhandlung von Schlossmann: über die sogenannte actio neg. gestorum ad exemplum institoriae actionis bekannt wurde. Gegen die Ausführungen Schlossmanns ist vor allen Dingen einzuwenden, dass denselben ausserordentliche Quellenwidrigkeit zu Grunde liegt, denn 1, l. 30 pr. D. neg. gest. ist nach seiner Behauptung corrumpirt. Er sagt S. 329: So wie die Stelle lautet, kann sie von Papinian nicht geschrieben sein. Der papinianische Text ist entweder von den Compilatoren interpolirt, oder in Folge eines Abschreibeversehens verändert. Als Beweis füh't er an, dass es nach seiner Auffassung der Rechtsgrundsätze eine solche Klage nicht geben kann; 2) die $1.5 \$ 3$ D. n. gest., in der Ulpian mit deutlichen Worten sagt, der procurutor eines Anderen habe ein Darlehen crhalten, beseitigt er S. 302, 303 mit der Bemerkung: das Darlehen von dem Ulpian rede, sei in Wirklichkeit ein Mandat. Dabei hat er dann viele Noth mit der Entscheidung Ulpians, dass dem Gläubiger die a. neg. gestorum gegen den Machtgeber zustehe. Er nimmt allerlei Voraussetzungen zu Hilfe, von denen die Stelle nichts sagt. 3) Ganz ähnlich verfährt er Note 15 mit Papinians l. 5 \& D. quando ex facto tutoris 26, 9. Papinian spricht hier von einem Vormund qui suo nomine mutuam pecuniam accepit und behandelt dabei auch den Fall, dass der creditor ideo contraxit, ut in causam iudicati pecunia transiret. „Auch hier, sagt Schlossmann, erfolgt die Hingabe des Geldes mit einer bestimmten Anweisung; das ist kein mutuum." Dass Papinian ausdrücklich sagt: mutuam pecuniam accepit, kümmert Schlossmann nicht. Interessanter wird das noch, wenn man die Entwickelung Papinians hier näher verfolgt. Er sagt nämlich, der Vormund habe Geld geliehen und mit dem Gelde eine Judicatsschuld des Pupillen bezahit. In diesem Falle habe der Gläubiger gegen den $\mathrm{Pu}$ pillen keine Klage. Anders verhalte es sich, wenn der Gläubiger bei der Contractschliessung ausgemacht habe, dass das geliehene Geld zur Bezahlung der Judicatsschuld zu verwenden sei. Es ist hier immer von demselben Contract die Rede, welchem in zweiten Fall noch eine Nebenberedung hinzutritt, und doch soll dieser Contract in dem zweiten Fall plötzlich zu einem Mandat geworden sein. Das wird für meine Behauptung der Quellenwidrigkeit wohl schon genügen. Wer in 
dieser Weise mit den Quellen umgeht, kann mit Leichtigkeit das ganze corpus iuris auf den Kopf stellen. Ich habe kürzlich noch einige Belegstellen für meine Auffassung gefunden, aber es kann ja natürlich nichts helfen, dass ich sie Schlossmann entgegenhalte. Er würde entweder sagen, dass der Text nicht echt sei, oder doch aus den Stellen das Gegentheil von dem herauslesen, was darin steht.

Was nun Schlossmanns Polemik gegen meine Ansicht betrifft, so bemerke ich zunächst, dass er dieselbe in mancher Hinsicht ganz falsch aufgefasst oder doch dargestellt hat, was besonders dadurch veranlasst ist, dass er den wesentlichsten Punkt ganz übersehen hat. Derselbe besteht darin, dass zur Begründung der Klage Papinians immer die in der Vollmacht zu findende Genehmigung des Machtgebers erforderlich ist. Eine reine Erfindung von ihm aber ist es, dass von mir ein "Seitenblick" des Dritten verlangt werde. Ich habe diesen Ausdruck nie gebraucht. Da die Ausführungen Schlossmanns trotz ilhrer Haltlosigkeit immerhin zu Missverständnissen führen könnten, so bemerke ich über meine Ansicht folgendes. A. giebt dem B. Vollmacht, 1000 Mark für ihn zu leihen. N. erklärt sich bereit, das Geld herzugeben. Aber weil B. und N. einfache Landleute sind, so wissen sie nichts davon, dass es einen Unterschied macht, ob B. in eigenem Namen oder in dem des A. contrahirt. Schlossmann scheint sich die contrahirenden Personen immer etwa so vorzustellen, als könnten sie ein juristisches Examen bestehen. B. contrahirt nun in eigenem Namen, wogegen N. nichts einzuwenden hat, aber da er nicht den B., sondern A. zum Schuldner haben will, so wird auf sein Verlangen ausdrücklich vereinbart, dass die $1000 \mathrm{Mark}$, welche B. schuldig geworden ist, nicht von B., sondern von A. dem Darlehnsgeber zurückzubezahlen sind. Schlossmann bemerkt S. 296: Wird jemand im Ernst glauben, dass eine Partei auf die Idee verfallen könnte, einen Darlehenscontract zu schliessen, um ihn sofort wieder aufzugeben und dadurch einen Gestionsanspruch zu gewinnen? Diese Frage ergiebt, dass Schlossmann das praktische Leben nicht kennt. Der mitgetheilte Fall zeigt dies und ich habe solchen Fall nicht einmal, sondern zehnmal erlebt. Freilich denkt der Gläubiger nicht 
daran, einen Gestionsanspruch zu erwerben, aber er will sein Geld nicht von dem Darlehensempfänger, sondern von dessen Machtgeber wiederfordern. Hat er nun aber gegen diesen eine Klage, obgleich der Bevollmächtigte nicht im Namen des Machtgebers, also nicht als Stellvertreter contrahirt hat? Diese Frage, mit welcher Schlossmann sich nicht befasst, so dass er an dem allerwesentlichsten Punkt dieser ganzen Lehre mit Stillschweigen vorbeigeht, hat mich veranlasst, mich überhaupt mit dieser Lehre zu befassen. Ich fand eine für den Gläubiger vollkommen genügende Rechtshülfe in den Grundsätzen der negotiorum gestio. Der Abschluss eines Contracts, zu dem jemand Vollmacht gegeben hat, ist ein Geschäft des Machtgebers und zwar ein im Voraus genehmigtes Geschäft. Wenn ein Dritter sich auf diese Contractschliessung einlässt und zwar lediglich desshalb, weil sie ein Geschäft des Machtgebers ist, wenn er also mit dem procurator feststellt, dass contemplatione domini contrahirt werde, so handelt er als Gessor des Machtgebers. Man vgl. z. B. l. 46 (45) pr. D. neg. gest.: - - et te eius voluntatis esse, ut emtam habere velles, agemus inter nos neg. gestorum. Er kann daher mit a. neg. gestorum seinen Schaden ersetzt verlangen. Dieser besteht in dem ganzen Darlehensbetrage, denn soviel hat er dadurch eingebüsst, dass er den Contract geschlossen, das Greschäft des Machtgebers also besorgt hat mit der, wie in dem mitgetheilten Falle, getroffenen Vereinbarung, dass nicht von dem Bevollmächtigten, sondern von dem Machtgeber Ersatz zu leisten sei. Der Nutzen des Geschäftsherm ist, wie ich gegen Schlossmann bemerke, auch dann vorhanden, wenn das Geld nach dem Empfange unterschlagen ist, denn ein genehmigtes Geschäft ist desshalb nützlich, weil es genehmigt ist. Die im Voraus oder nachträglich ertheilte Genehmigung entscheidet endgültig darüber, dass das Geschäft das Interesse des Geschäftsherrn berührt ${ }^{1}$ ).

1) Man vergleiche über diese Punkte indess auch Kohler, Jahrb. für Dogm. Bd. 25 S. 127, der es viel besser versteht, den Bedürfnissen des Verkehrs gerecht zu werden, als Schlossmann. Doch scheint auch Kohler zu übersehen, dass ich zur Begründung der Klage Papinians die in der Vollmacht liegende Genehmigung der neg. gestio des Dritten verlange. 
Papinian nennt die Klage in diesem Falle: actio neg. gestorum ad exemplum institoriae, weil sie wie die eigentliche institoria auf der Vollmacht beruht. Er hat damit keine eigenartige a. neg. gestorum geben, sondern nur ausdrücken wollen, dass die Klage in diesem Falle dieselbe Wirkung hat, wie die eigentliche institoria, weil sie wie die letztere auf der Vollmacht beruht. Dass der procurator Vollmacht hatte, als er contrahirte, ist, wie ich noch einmal wiederhole, zur Begründung der von Papinian gegebenen Klage immer nothwendig. Die Klage wäre auch vorhanden, wenn wir die 1. 30 pr. D. neg. gest. nicht hätten, denn sie folgt aus den Grundsätzen der neg. gestio.

Meine Auffassung unterscheidet sich, in Ansehung des praktischen Erfolges, von der herrschenden besonders in folgendem Punkte. Nach der letzteren kann der Dritte nur dann gegen den Machtgeber klagen, wenn der Bevollmächtigte im Namen des Machtgebers contrahirt hat. Nach meiner Ansicht hat er die Klage gegen den Machtgeber auch in dem Fall, wo der Bevollmächtigte in eigenem Namen contrahirt, daneben aber mit dem Dritten vereinbart hat, dass die Verbindlichkeit nicht von ihm, sondern von seinem Machtgeber zu erfüllen sei. Diese Vereinbarung liegt auch dann vor, wenn neben der Contractschliessung ausgemacht ist, dass der Gegenstand des Vertrages, z. B. der Betrag, welchen der Bevollmächtigte in eigenem Namen geliehen hat, lediglich für die vollmachtgemässen Zwecke des Machtgebers bestimmt sei. Hat ein Vormund oder Bevollmächtigter Geld gelielıen und ist dabei von dem Gläubiger ausbedungen, dass der geliehene Betrag zur Bezahlung einer Schuld des Geschäftsherrn zu verwenden sei, so ist damit festgestellt, dass der Betrag lediglich von dem Geschäftsherrn zu erstatten ist. L. $6 \S 1$ (l. 5 \$ 3$)$ D. neg. gest. und $1.5 \S 1$ D. quando ex facto tutoris. Schlossmann sieht in dieser Auslegung des Parteiwillens Fictionen, weil er es überhaupt nicht versteht, sich in den Gedankengang gewöhnlicher Menschenkinder hineinzufinden. Er selbst würde freilich ganz anders verfahren, denn er würde als Bevollmächtigter im Namen seines Machtgebers contrahiren. Aber es kommt darauf an, wie die Personen verfahren, welche von den rechtlichen Grundsätzen nichts wissen. Auf solche Personen, 
welche doch die überwiegende Mehrheit bilden, nimmt Schlossmann keine Rücksicht.

Was nun endlich die Polemik betrift, welche Schlossmann sonst noch gegen mich führt, so brauche ich ihm darin nicht zu folgen. Wenn man beachtet, dass ich zur Begründung der institorischen actio neg. gestorum die Bevollmächtigung des Vertreters voraussetze, so wird man finden, dass alle die theoretischen Abgründe, welche Schlossmann sich ausgedacht hat, in nichts zerfallen. Doch mag um seine Polemik zu kennzeichnen, ein kleiner Punkt erwähnt werden. In $1.30 \mathrm{pr}$. D. neg. gest. sagt Papinian auch, dass der Gläubiger einen Bürgen angenommen habe und Schlossmann deducirt nun, dass ja der Gläubiger den Bürgen gleich wieder verloren habe, wenn die Darlehensforderung gleich von ihm aufgegeben sei, denn der Bürge sei natürlich für die Darlehensschuld des Mandatars bestellt. Schlossmann hält die Stelle für unecht, aber soweit sie für seine Meinung günstig zu sein scheint, ist sie natürlich echt. Uebrigens ist seine Auffassung in Ansehung des Bürgen falsch. Die Stelle ergiebt, dass der Gläubiger contrahirt hat, mit der Absicht, den Mandanten zum Schuldner $z u$ erhalten (cujus litteras secutus). Es ist daher zweifellos, dass der Bürge für die Schuld des Mandanten verlangt und bestellt ist.

Zum Schluss noch folgende Frage. Wenn heute Jemand einem Bevollmächtigten, der in eigenem Namen contrahirt, $1000 \mathrm{M}$. gegen $4 \%$ Zinsen leiht, dabei aber ausmacht, dass der geliehene Betrag zur Bezahlung einer Schuld des Machtgebers zu verwenden sei - ist man dann zu der Annahme berechtigt, dass dem Bevollmächtigten lediglich der Auftrag gegeben sei, die $1000 \mathrm{M}$. zur Bezahlung der Schuld des Machtgeber's zu verwenden und dass also weder ein Darlehensvertrag noch ein Zinsversprechen vorliege? Wenn man dazu nicht berechtigt ist, so hat man ebensowenig das Recht, in den Fällen der $1.5 \S 3$ D. neg. gest. und 1.5 \& 1 D. quando ex facto tutor, den Darlehensvertrag aus dem Grunde zu leugnen, weil daneben eine Vereinbarung über die Verwendung des Geldes getroffen ist. Wenn Schlossmann etwa meint, dass so etwas heute nicht vorkommt, so zeigt er damit wieder, dass er das praktische Leben nicht kennt. 
344 E. Ruhstrat, Ueber đie römischen Handlungsbevollmäehtigten.

Da Schlossmann sich S. 310 auf das Wort beruft: ,an ihren Früchten sollt ihr sie erkennen", so will ich in Beziehung auf die Früchte seiner Auffassung der Quellen doch noch folgendes Beispiel mittheilen. Der Handlungsbevollmächtigte eines Krämers kauft in eigenem Namen Waaren von einem Grosshändler oder von dessen Reisenden, aber auf Verlangen des Verkäufers wird dabei verabredet, dass die verkauften Waaren in dem Geschäft des Krämers zur Verwendung $\mathrm{zu}$ bringen sind. Die Praxis würde in diesem Falle unbedenklich feststellen, dass die Umstände ergeben, es sei nach dem Willen der Contrahenten im Namen des Machtgebers also des Krämers contrahirt. Ich würde mich dafür entscheiden, dass dem Verkäufer die Klage Papinians zu geben sei. Schlossmann würde den Contract ebenso aufzufassen haben wie die 1.6 \& 1 (I. 5 \& 3) D. neg. gest. Er würde folglich sagen, dass der Verkäufer den Handlungsdiener des Krämers bea uftragt habe, die Waaren in dem Geschäfte des Krämers zu verkaufen. Der Grosshändler würde also die Geschäfte des Krämers durch dessen Handlungsdiener besorgen lassen. Seltsame Verirrung! Die eben genannte Stelle ist aus dem Grunde nicht selten missverstanden, weil man es für ausgemacht hielt, dass die adjecticische Doppelhaft auf die Contractschliessungen der Procuratoren ausgedehnt sei, während Ulpian in der Stelle so deutlich wie möglich sagt, dass diese Ausdehnung nicht stattgefunden habe. Denn der procurator, welcher unter vertragsmässiger Bezugnahme auf den Machtgeber mit einem Dritten contrahirt hat, kann nach Ulpians ausdrücklichen Worten von dem Dritten nicht verklagt werden (cum quo contraxi nullam). Ich werde auf diesen Grundsatz und dessen Folgen noch ausführlich zurückkommen. Bei dieser Gelegenheit werde ich dann auch wohl noch näher auf die Schlossmannschen Irrthümer zurückkommen. 